\title{
ANMERKUNGEN ZUR GESTE IM FRÜHEN FILM
}

\author{
FranK KeSSLER
}

\begin{abstract}
Als Maksim Gor'kij 1896 in einem Lokal in Nižnij-Novgorod einer Vorstellung des Cinématographe Lumière beiwohnt, wähnt er sich, wie er in einem Zeitungsartikel schreibt, »im Reich der Schatten«.
\end{abstract}

m) Und alles lautlos, schweigend, absonderlich. Man hört nicht das Rumpeln der Räder auf dem Straßenpflaster, nicht das Trappeln der Schritte, keine Stimme, nichts - nicht eine einzige Note jener vielschichtigen Symphonie, die immer die Bewegung von Menschen begleiten. Lautlos wiegt sich das aschgraue Laub der Bäume im Wind, lautlos gleiten die grauen, schattengleichen Figuren der Menschen über die graue Erde, wie von einem Fluch zum Schweigen Verdammte und grausam Bestrafte, denen man alle Farben des Lebens genommen hat. Ihr Lächeln ist tot, obwohl ihre Bewegungen voll lebendiger Energie und so schnell sind, dass man sie kaum erfassen kann. Ihr Lachen ist tonlos, obwohl Sie sehen, wie die Muskeln in den grauen Gesichtern zucken. [...] Da - ein neues Bild: Am Tisch sitzen drei Kartenspieler. Angespannte Gesichter, schnelle Bewegungen der Hände beim Kartenausteilen. Am Zittern der Hände wie an der Muskelanspannung der Gesichter sieht man die Gier der Spieler. Das Spiel läuft ... und sie beginnen alle zu lachen, und es lacht auch der Kellner, der Bier bringt und an ihrem Tisch stehen bleibt. Sie krümmen sich vor Lachen ... aber kein Laut ist zu hören. Als wären diese Menschen gestorben und ihre Schatten dazu verurteilt, bis in alle Ewigkeit schweigend Karten zu spielen.« (Pacatus 1995: 13f.)

Das gutbürgerliche Vergnügen der PARTIE D'ÉCARTÉ auf dem südfranzösischen Landsitz der Brüder Lumière, an dem deren Vater Antoine Lumière, der Magier und Schausteller Félicien Trewey sowie der Brauer Alphonse Winckler teilnehmen, wird bei Gor'kij zu einem gespenstischen Totentanz. Nun ist Gor'kij gewiss eine dissonante Stimme im Chor der zeitgenössischen Kommentatoren, die angesichts der Lumière-Filme in ihrer Mehrzahl bekanntlich ganz im Gegenteil eher geradezu synästhetische Wahrnehmungserfahrungen schildern. Dennoch ist die von ihm beschriebene Wirkung der lebenden Bilder, die, wie er schreibt, »nicht das Leben, sondern [den] Schatten des Lebens « zeigen, in besonderer Weise geeignet, den Status der Geste im frühen Kino zu reflektieren. 
In Gor'kijs Beschreibung - wie kritisch sie gegenüber den kinematographischen Bildern auch immer sein mag - wird die körperliche Bewegung als Ausdrucksbewegung gelesen, gleichzeitig aber von jeglicher Körperlichkeit losgelöst. Die Menschen sind nichts weiter als Schatten, und dennoch verrät die »Muskelanspannung der Gesichter [...] die Gier der Spieler«. Das Verhalten der Personen wird, mit anderen Worten, hier selbst zur Geste, auf ähnliche Weise wie Béla Balázs mehr als ein Vierteljahrhundert später in DER SICHTBARE MENSCH (1924) feststellt, das Sprechen im Stummfilm zur »Sprachgebärde« wird (Balázs 1982: 69). Die hier skizzierte Linie ließe sich - geradezu kurioserweise - ohne weiteres verlängern zu einer Auffassung vom Film als »behavioristische« Kunstform, wie man sie bei Maurice Merleau-Ponty weitere zwanzig Jahre später findet. In dem 1945 gehaltenen Vortrag »Das Kino und die neue Psychologie« heißt es:

"Das Kino zeigt uns nicht, wie es der Roman lange getan hat, die Gedanken des Menschen, es zeigt uns sein Benehmen oder sein Verhalten, es bietet uns unmittelbar diese besondere Weise des Zur-Welt-seins, die Dinge und die Anderen zu behandeln, die für uns in den Gesten, dem Blick, dem Mienenspiel sichtbar ist und die offensichtlich jede uns bekannte Person definiert. Wenn uns das Kino eine Person zeigen will, der schwindlig ist, wird es nicht versuchen müssen, die innere Landschaft des Schwindels wiederzugeben [...]. Wir werden den Schwindel viel besser empfinden, indem wir ihn von außen sehen, indem wir diesen aus dem Gleichgewicht geratenen Körper betrachten, der sich an einer Steilklippe dreht, oder diesen schwankenden Gang, der versucht ist, sich man weiß nicht auf welchen Umsturz des Raums einzustellen. Für das Kino sind Schwindel, Freude, Schmerz, Liebe, Haß Verhaltensweisen wie für die moderne Psychologie.« (Merleau-Ponty 1997: 244f)

Für Gor'kij, wie dann später für Balázs und Merleau-Ponty, wird gefilmtes Verhalten somit zur Ausdrucksbewegung, die zum Ausdruck gebrachte Emotion prägt sich dem Körper gleichsam auf, ob dieser selbst nun als Schatten erscheint oder, ganz im Gegenteil, in seinem In-derWelt-sein. Dies jedoch ist, genauer betrachtet, letztlich nur darum möglich, weil dieses Verhalten in eine, wie immer auch rudimentäre, narrative Struktur eingebettet ist. Sowohl Gor'kij als auch Merleau-Ponty (und letztlich selbst Balázs, allerdings an dieser Stelle nur implizit) schildern kurze narrative Sequenzen - das Kartenspiel, der Mensch, dem an einer Steilklippe schwindlig wird -, durch die das Verhalten ein gerichtetes, aus der Situation heraus erklärbares wird. Damit ließe sich in der Tat eine Entwicklungslinie ziehen, in deren Verlauf das Kino von einer unwirklichen Schattenwelt zu einer behavioristischen, und somit auch realistischen Kunstform wird, weil es sowohl in stilistischer wie in technologi- 
scher Hinsicht einen immer stärkeren Wirklichkeitseindruck bewirkt. In einer solchen teleologischen Perspektive wäre es also geradezu die $\mathrm{Be}$ stimmung des kinematographischen Apparats, die Alltagsgestik einzufangen, womit umgekehrt das alltägliche Verhalten letztlich die ästhetische Norm für das gestisches Spiel des Filmschauspielers setzte.

Ganz anders als der Zeitgenosse Gor'kij beurteilt nun der französische Filmhistoriker Georges Sadoul PARTIE D'ÉCARTÉ, der diesen Film auf eine Weise beschreibt, die in dessen Innern das Aufscheinen einer anderen Form der Gestik sichtbar macht, und sei es in diesem Fall auch nur als eine Art potentieller Störfaktor:

»Drei Kartenspieler, alle eher stattlich, die großen Füße fest auf den Boden gestemmt, füllen die Leinwand. Hinter ihnen eingetopfte Palmen. Ein Spieler teilt die Karten aus, sogleich beginnt die Partie. Ein Kammerdiener bringt Bier, das in den Gläsern schäumt. Einer der Spieler, der Brauer A. Winkler, hat das Bier ausgeschenkt. Antoine Lumière, der seinem Freund Trewey gegenüber sitzt, zündet eine Zigarre an und raucht einige Züge. Schon ist die Partie beendet. Einer der Spieler nimmt sich den Einsatz. Der Kammerdiener streicht die ganze Zeit lachend und mit theatralischen Gesten um die Spieler herum, die Arme nach oben werfend und sich auf die Schenkel klopfend. Doch die Anwesenheit dieser ein wenig künstlichen Figur - welche die kleinen Nebenrollen in den Filmen von Méliès ankündigt - kann die Natürlichkeit dieser schnell gespielten Partie nicht stören, ein gutbürgerlicher Zeitvertreib an einem sonnigen Sonntag.« (Sadoul 1973: 291f)

Sadoul schildert somit einerseits den Effekt des eingefangenen Lebens, der, einer oft zitierten Formel zufolge (»la vie pris sur le vif«), die Lumière-Filme charakterisiert, andererseits aber bricht hier, in der Person des Kammerdieners, die Theatralität ein in die Natürlichkeit der Szene. Der Mann, dessen Verhalten in einer Fußnote mit seinem südfranzösischen Naturell erklärt wird, ist, wenn man Sadoul wörtlich nimmt, eigentlich im falschen Film - er gehört eher in das kinematographische Universum der inszenierten Filme von Georges Méliès als in das der Brüder Lumière. Das Adjektiv »theatralisch« steht für einen ganzen Komplex negativer Zuschreibungen an diesen Auftritt: Theatralisch ist zu lesen als »nicht filmgemäß«, als »nicht natürlich«, als »übertrieben«; der Term bezeichnet somit letztlich etwas, das der Film zu überwindenden hat, um wirklich Film zu werden.

Im Kern ist damit auch die - zumindest außerhalb des Bereichs der Filmhistoriker, die sich dem frühen Film widmen - vorherrschende Haltung zur Gestik in der Frühzeit umschrieben: Das Kino, das seine Form erst noch finden muss, ist in seiner "primitiven « Phase noch zu sehr dem Theater verhaftet und kann sich erst im Laufe der Jahre, in denen der 
Film tatsächlich zur Kunst wird, aus dieser Verbindung lösen. Die Gestik ist dementsprechend noch »theatralisch $\ll$ im Sinne der verschiedenen $\mathrm{Be}$ deutungsschichten dieses Ausdrucks.

Historisch betrachtet ist eine solche Perspektive jedoch überaus problematisch, und zwar nicht nur weil, wie Lea Jacobs und Ben Brewster (1997 und 1998) oder auch Roberta Pearson (1992) in ihren detaillierten Studien nachgewiesen haben, das Spiel der Darsteller im Kino nur um den Preis einer überaus simplistischen Sicht der Dinge so umstandslos mit der zeitgenössischen Theaterpraxis über einen Kamm geschoren werden kann: Diese Sichtweise wird weder den komplexen stilistischen Normen des Bühnenspiels, noch der gestischen Arbeit der frühen Filmschauspieler gerecht. Unzureichend ist diese Perspektive vor allem aber auch, weil sie ausgeht von einer negativen, ahistorischen und teleologischen Betrachtungsweise: Der frühe Film - und insbesondere die Spielweise der Darsteller - entspricht nicht den ästhetischen Normen dessen, was der Film einmal sein wird. Und gleichzeitig nimmt sie die Tatsache nicht zur Kenntnis, dass die Kinematographie der Frühzeit doch ganz offensichtlich ihr Publikum zu erreichen wusste, dass sie nicht gesehen wurde als ein Medium mit einem grundlegenden Mangel, sondern dass sie nur in ihrem historischen Funktionieren zu verstehen ist, das heißt, wenn man die ihr inhärente Logik begreift.

Das, was mit Blick auf den frühen Film nun so unzutreffend mit dem Begriff »theatralisch« bezeichnet wird, meint eigentlich vor allem Folgendes:

- die Gestik des frühen Films sucht plakative Eindeutigkeit und stützt sich dabei auf die konventionellen Darstellungsformen eines gestischen Repertoires, die in gewissen Formen der Pantomime, der kommunikativen Alltagsgestik sowie in vielerlei zeitgenössischen Schauspiel-Lehrbüchern zum Körperausdruck kodifiziert sind;

- die Gestik richtet sich nicht an die anderen Figuren, sondern vor allem an das Publikum und strebt nicht danach, eine diegetische Kommunikationssituation bzw. Gefühlswelt realistisch darzustellen; sie signalisiert Emotionen, Intentionen, Reaktionen mehr als dass sie als deren Ausdruck verstanden werden könnte;

- die Darstellungsweise bekümmert sich nicht darum, die Illusion eines geschlossenen diegetischen Raums zu schaffen, sondern sie richtet sich immer wieder hin zur Kamera und damit auch an das Publikum. ${ }^{1}$

1 Vgl. zu diesen Punkten auch Kessler/Lenk 1995, 1996 und 1997. 
All diese Aspekte sollten jedoch nicht als Residuen eines filmfremden, anderen medialen Formen zugehörigen Stils angesehen werden, den das Kino zu überwinden hatte, um gewissermaßen »zu sich selbst zu finden«. Sie gehören schlicht einem anderen ästhetischen Paradigma an, einem anderen Repräsentationsmodus, und entsprechen letztlich recht genau den Merkmalen, die Tom Gunning dem »Kino der Attraktionen« zuschreibt, das in seinem Funktionieren dem klassischen Erzählkino geradezu Punkt für Punkt entgegengesetzt ist:

»Was genau ist nun das Kino der Attraktionen? Zunächst einmal ein Kino, das sich auf die von Léger beschriebene Eigenschaft beruft: die Fähigkeit, etwas zu zeigen. Im Gegensatz zum voyeuristischen Aspekt des narrativen Kinos, wie er von Christian Metz analysiert wurde, ist es ein exhibitionistisches Kino. Ein Aspekt des frühen Films [...] ist charakteristisch für das ganz andere Verhältnis, das das Kino der Attraktionen zum Zuschauer herstellt: Die Schauspieler blicken immer wieder direkt in die Kamera. Dieses Mittel, das später als der Realismus-Illusion abträglich gelten sollte, wird hier noch mit großer Emphase eingesetzt und dient der Kontaktaufnahme mit dem Publikum. Von den Komikern, die für die Kamera Grimassen schneiden, zum ständigen Verbeugen und Gestikulieren der Magier in Zauberfilmen, ist dies ein Kino, das seine Sichtbarkeit zur Schau stellt und gerne bereit ist, eine in sich geschlossene fiktive Welt aufzubrechen, wenn ihm dies die Chance eröffnet, die Aufmerksamkeit des $\mathrm{Zu}$ schauers zu beanspruchen.« (Gunning 1996: 27)

Wenn nun das Kino der Attraktionen, wie Gunning schreibt, in erster Linie »seine Sichtbarkeit zur Schau stellt«, statt ein geschlossenes diegetisches Universum zu erschaffen, so kommt der Gestik hier in der Tat eine andere Funktion zu als im klassischen narrativen Kino. Zielt sie in letzterem vor allem darauf ab, dem Verhalten oder den Gefühlen der Figur Authentizität, Natürlichkeit oder Glaubwürdigkeit in der jeweiligen narrativen Situation zu verleihen, hat sie in der Frühzeit die Aufgabe, je nach Kontext eine Situation, eine Intention, eine Reaktion oder eine Emotion für den Betrachter eindeutig zu charakterisieren. Dabei kann sie zwar immer noch narrativ funktional sein, doch gleichsam auf einer Art Metaniveau: Einerseits ereignet sie sich zwangsläufig innerhalb des jeweiligen diegetischen Raum, wobei sie andererseits aber über diesen hinausweist, indem die Gestik den Zuschauern zugewandt ist und eher auf das Erleben der Figuren hindeutet, anstatt ihm in einer den Normen des realistischen Darstellungsstils entsprechenden Weise Ausdruck zu verleihen.

In den Filmen von Georges Méliès finden sich dazu zahllose Beispiele, von hinweisenden Gebärden, die dem Betrachter zeigen, dass sich etwas Erstaunliches oder Spektakuläres ereignet bzw. gleich ereignen wird, über die gestische Kommunikation mit dem Publikum, die Méliès auf be- 
sonders eindrucksvolle Weise in LES CARTES vIVANTES (1904) darstellt und dabei mit seiner Gestik selbst angebliche Reaktionen und Zurufe der Zuschauer gewissermaßen spiegelt, bis zu den vielfältigen die Handlung oder die auftretenden Figuren kommentierenden Gesten, wie z. B. die vor dem Gesicht kreisende Handbewegung, mit der eine Frau als schön charakterisiert wird (vgl. Kessler/Lenk 1997).

Schließlich kann so die Gestik selbst zur Attraktion werden, ihrerseits einen spektakulären Charakter erhalten und in den Mittelpunkt des Geschehens rücken. Dies geschieht insbesondere in vielen komischen Filmen, wenn die Figuren zu Opfern irgendwelcher äußeren Einflüsse werden, was sich dann in ihrem Verhalten und ihren Gebärden niederschlägt. Ein Beispiel unter vielen ist der Film IL NATALE DI CRETINETTI (auch bekannt unter seinem französischen Titel LES TROIS FLACONS DE GrIBOUILLE) aus dem Jahr 1909, mit dem französischen Komiker André Deed in der Hauptrolle. Hier sind es die Dämpfe, die drei zerbrochenen Flaschen entweichen, die bei den Bewohnern eines Hauses abwechselnd Frohsinn, Angst oder Wut auslösen, was sich dann aus ihrer jeweiligen Mimik und Gestik ablesen lässt. Diese selbst wird nun, nicht zuletzt wegen der vielfältigen Variationen des Gefühlsausdrucks bei den verschiedenen Darstellern, zur komischen Attraktion, während die narrative Situation sich nicht mehr weiter entwickelt, nachdem die Dämpfe ausgetreten sind und Zug um Zug das gesamte Haus durchdringen (vgl. Kessler/Lenk 1996).

Wenn für Maksim Gor'kij also der Kinematograph ein Reich der Schatten präsentiert, in dem die Gesten körperloser Wesen dem Betrachter ein Schauspiel unnatürlichen Lebens darbieten, so liest er die Gestik in erster Linie mit Blick auf ihre Alltagsbedeutung, als einen Aspekt der menschlichen Existenz. Die Medialisierung erscheint bei ihm lediglich als Entwirklichung oder, genauer, als Entkörperlichung der Welt. Für die große Mehrheit seiner Zeitgenossen jedoch zeigt die Kinematographie vor allem visuelle Attraktionen, wobei die Gestik selbst Teil des spektakulären Schauspiels ist, ob sie nun als la vie prise sur le vif oder als inszenierte Darstellung vorgeführt wird. Der Kammerdiener Louis Lumières, so könnte man sagen, scheint dies begriffen zu haben: Er präsentiert sich nämlich, »die Arme nach oben werfend und sich auf die Schenkel klopfend«, ganz unumwunden als Attraktion. 


\section{Literatur}

Balázs, Béla (1982): Schriften zum Film. Bd.1: >Der sichtbare Menschく. Kritiken und Aufsätze 1922-1926, München: Carl Hanser Verlag. Brewster, Ben/Jacobs, Lea (1997): Theater to Cinema, Oxford: Oxford University Press.

Brewster, Ben/Jacobs, Lea (1998): »Piktorialer Stil und Schauspiel im Film«. KINtop. Jahrbuch zur Erforschung des frühen Films 7, S. 3762.

Gunning, Tom (1996): »Das Kino der Attraktionen«. Meteor 4, S. 25-34. Kessler, Frank/Lenk, Sabine (1995): »...levant les bras au ciel, se tapant sur les cuisses. Réflexions sur le geste dans le cinéma des premiers temps«. In: Roland Cosandey/François Albera (Hg.), Cinéma sans frontières 1896-1918. Images Across Borders, Lausanne: Payot/ Québec: Nuit Blanche, S.133-145.

Kessler, Frank/Lenk, Sabine (1996): »L'expression des sentiments dans la comédie des premiers temps«. La licorne 37, S.7-15.

Kessler, Frank/Lenk, Sabine (1997): »L'adresse-Méliès«. In: Jacques Malthête/Michel Marie (Hg.), Georges Méliès, l'illusionniste fin de siècle?, Paris: Presses de la Sorbonne Nouvelle, S.183-199.

Merleau-Ponty, Maurice (1997): »Das Kino und die neue Psychologie«. In: Ralf Konersmann (Hg.), Kritik des Sehens, Leipzig: Reclam, S. 227-246.

Pacatus, I. M. [Maksim Gor'kij] (1995): »Flüchtige Notizen«. KINtop. Jahrbuch zur Erforschung des frühen Films 4, S. 13-16.

Pearson, Roberta (1992): Eloquent Gestures. The Transformation of Performance Style in the Griffith Biograph Films, Berkeley/Los Angeles/Oxford: University of California Press.

Sadoul, Georges (1973): Histoire générale du cinéma, Bd. 1, Paris: Denoël. 